\title{
Leprosy in Togo: retrospective study of 2630 cases over 15 years
}

\section{Koussake Kombate ${ }^{1,2}$, Julienne Noude Teclessou, ${ }^{1,2}$, Bayaki Saka, ${ }^{1,2}$, Gora Lucienne Tabe-Djato', Abla Séfako Akakpo ${ }^{1,2}$, Abas Mouhari-Toure ${ }^{3,4}$, Palokinam Pitche ${ }^{1,2}$}

${ }^{1}$ Department of Dermatology, Teaching Hospital of Lomé, Lomé, Togo , ${ }^{2}$ Department of Health Sciences, University of Lomé, Lomé, Togo, ${ }^{3}$ Department of Dermatology, Teaching Hospital of Kara, Kara, Togo, ${ }^{4}$ Department of Health Sciences, University of Kara, Kara, Togo

Corresponding author: Dr. Julienne Noude Teclessou, E-mail: tjulie06@yahoo.fr

\begin{abstract}
Objective: The aim of this study was to determine the evolutionary trends, the clinical forms of leprosy cases notified in Togo and to map them. Methods: This was a retrospective study from 1 January 2000 to 31 December 2014 on all leprosy cases notified by all districts to the national leprosy control program (PNLL). Results: A total of 2,630 new cases of leprosy were notified. The mean annual incidence was 175.33 cases with extremes of 73 to 266 cases. The median age of patients was 40 years, and the sex ratio was 1.13 . Children aged 0 to 15 accounted for $5.5 \%$ of the cases. The plateau region in the center of the country had the highest annual incidence (around 800 cases). Clinically, multibacillary forms accounted for $74.2 \%$ of cases. All patients were treated with multidrug therapy (MDT). The WHO grade 2 disease was found in $15 \%$ of patients and $9.5 \%$ were lost to follow-up. Conclusion: Our results confirm the elimination of leprosy in Togo. There is a high incidence of leprosy in some areas of the country, which may raise fears of a resurgence of the disease
\end{abstract}

Key words: Epidemiology, leprosy, Lome

\footnotetext{
How to cite this article: Kombate K, Teclessou JN, Saka B, Tabe-Djato GL, Akakpo AS, Mouhari-Toure A, Pitche P. Leprosy in Togo: retrospective study of 2630 cases over 15 years. Our Dermatol Online. 2017;8(Suppl. 1):10-14.

Submission: 22.09.2017; Acceptance: 28.10.2017

DOI: 10.7241 /ourd.2017s.3
} 


\section{Lèpre au Togo: étude rétrospective de 2630 cas sur 15 ans}

\section{Koussake Kombate ${ }^{1,2}$, Julienne Noude Teclessou, ${ }^{1,2}$, Bayaki Saka, ${ }^{1,2}$, Gora Lucienne Tabe-Djato', Abla Séfako Akakpo ${ }^{1,2}$, Abas Mouhari-Toure ${ }^{3,4}$, Palokinam Pitche ${ }^{1,2}$}

${ }^{1}$ Department of Dermatology, Teaching Hospital of Lomé, Lomé, Togo , ${ }^{2}$ Department of Health Sciences, University of Lomé, Lomé, Togo, ${ }^{3}$ Department of Dermatology, Teaching Hospital of Kara, Kara, Togo, ${ }^{4}$ Department of Health Sciences, University of Kara, Kara, Togo

Corresponding author: Dr. Julienne Noude Teclessou, E-mail: tjulie06@yahoo.fr

\section{RÉSUMÉ}

Objectif: Le but de cette étude était de déterminer les tendances et les formes cliniques de lèpre notifiées au Togo. Méthode: Il s'agissait d'une étude rétrospective du 1 er janvier 2000 au 31 décembre 2014 portant sur les cas de lèpre notifiés par l'ensemble des districts sanitaires au programme national de lutte contre la lèpre (PNLL). Résultats: Au total, 2630 nouveaux cas de lèpre avaient été notifiés. Lincidence annuelle moyenne était de 175,33 cas avec des extrêmes de 73 à 266 cas. Lâge médian des patients était de 40 ans, et le sex-ratio de 1,13. Les enfants âgés de 0 à 15 ans représentaient $5,5 \%$ des cas. La région des plateaux dans le centre du pays cumulait l'incidence annuelle le plus élevé (environs 800 cas). Sur le plan clinique, les formes multibacillaires représentaient 74,2\% des cas. Tous les patients étaient traités par polychimiothérapie. Linfirmité grade 2 de l'OMS était retrouvée chez 15\% des patients et 9,5\% étaient perdus de vue. Conclusion: Nos résultats confirment l'élimination de la lèpre au Togo. On note une incidence élevé de la lèpre dans certaines régions du pays pouvant faire craindre une recrudescence de la maladie.

Key words: Epidémiologie; Lèpre; Lomé

\section{INTRODUCTION}

La lèpre est une maladie infectieuse causée par Mycobacterium leprae. Au niveau mondial, elle occupe, après la tuberculose, le deuxième rang des infections humaines dues à des mycobactéries. Il s'agit d'une maladie ayant sévit pendant plusieurs décennies de façon endémique dans plusieurs pays d'Afrique. La stabilité de l'incidence de la lèpre au cours de la dernière décennie témoigne d'une transmission toujours active de la bactérie. En effet, le nombre de nouveaux cas dépistés dans le monde de 2005 à 2015 varie de 299036 à 210740 [1]. En Afrique, ce nombre varie entre 45179 et 20004 [1]. Au Togo, la lèpre n'est plus un problème prioritaire de santé publique depuis plusieurs années [2].

Le but de cette étude était de déterminer les tendances et les formes cliniques des cas de lèpre notifiés au Togo.

\section{MATERIALS AND METHODS}

Il s'agissait d'une étude rétrospective menée de janvier 2000 à décembre 2014 portant sur tous les cas de lèpre notifiés sur l'ensemble des six districts sanitaires du Togo. Le Togo est un pays d'Afrique de l'Ouest qui compte environs 7500.000 d'habitants en 2014 [3]. On y dénombre 6 régions sanitaires du nord vers le sud: la région des savanes, la région de la Kara, la région centrale, la région des plateaux, la région maritime et la région de Lomé commune. Tous les cas de lèpre documentés et traité entre 2000 et 2014 par le programme national de lutte contre la lèpre (PNLL) ont été inclus dans l'étude. Les cas de lèpre ont été classés en paucibacillaires ou multibacillaires selon la classification clinique de l'Organisation Mondiale de la Santé (OMS) [4].

\footnotetext{
How to cite this article: Kombate K, Teclessou JN, Saka B, Tabe-Djato GL, Akakpo AS, Mouhari-Toure A, Pitche P. Lèpre au Togo: étude rétrospective de 2630 cas sur 15 ans. Our Dermatol Online. 2017;8(Suppl. 1):10-14.

Submission: 22.09.2017; Acceptance: 28.10.2017

DOI: 10.7241 /ourd.2017s.3
} 
La collecte des données a été faite à partir d'une fiche d'enquête préalablement établie. Les variables étudiées étaient: les données démographiques (nom, prénom, âge, sexe), les données cliniques (les formes de lèpre, le degré d'invalidité selon l'OMS), les données sur le l'issue de la maladie. Nous avons ensuite calculé les indicateurs d'élimination. Le taux de détection correspond au nombre total de nouveaux cas de lèpre dépistés durant l'année rapporté à celui de la population totale exprimée en taux pour 1000 habitants.

Lanalyse des données a été fait avec le logiciel STATA/ Statall et logiciel Health Mapper

\section{RESULTS}

De 2000 à 2014, 2630 nouveaux cas de lèpre ont été enregistrés sur l'ensemble du pays. L'incidence annuelle moyenne était de 175,33 nouveaux cas par an avec des extrêmes de 73 et 266 cas.

Lâge médian des patients était de 40 ans avec des extrêmes de 2 ans à 96 ans. Les formes pédiatriques ( 0 à 15 ans)représentaient 5,6\%. La sex-ratio (M/F)était de 1,13.

L'incidence annuelle des cas varie de 73 en 2014 à 266 en 2001 avec des pic de 260 en 2009 et 258 en 2000. Le taux d'incidence de la lèpre varie de 6,1 cas pour 100000 habitants en 2000 à 1,2 cas pour 100000 habitants en 2014. Ce taux d'incidence diminue progressivement de 6,1 en 2000 jusqu'à 3,3 pour 100000 en 2004; une stagnation du taux d'incidence entre 2004 et 2008 puis une évolution en dent de scie entre 2008 et 2012 (Fig. 1).

Sur le plan clinique, toutes les formes de la lèpre étaient observées. Selon la classification de l'OMS, 1951 patients $(74,2 \%)$ avaient une forme multibacillaire (MB)contre 679 patients $(25,8 \%$ ) qui avaient une forme paucibacillaire $(\mathrm{PB})$. Lincidence annuelle des cas des formes MB varie de 57 en 2014 à 210 en 2009 avec un pic de 194 en 2001. Lincidence annuelle des cas des formes $\mathrm{PB}$ varie de 16 en 2014 à 85 en 2000 et en 2002 (Fig. 2).

En fonction des régions, l'incidence de la lèpre reste plus élevée dans la région des plateaux dans le centre du pays (600 cas de forme MB et 200 cas de forme PB) suivie de la régions de Kara (300 cas de forme MB et 100 cas de formes $\mathrm{PB}$ )et de la région des savanes (environs 300 cas de forme MB et 100 cas de formes PB) (Fig. 3).

Tous les patients étaient traités par la PCT. Sur les 2630 patients colligés, 2316 (88,06\%)avaient achevés le

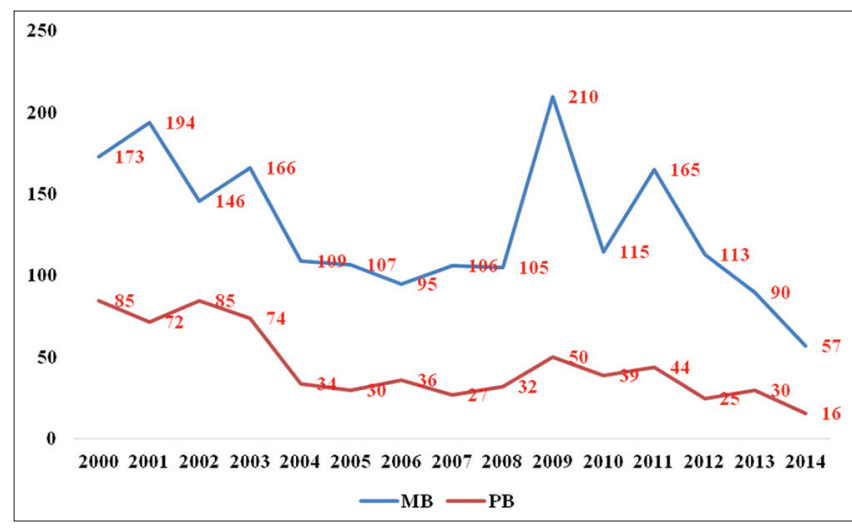

Figure 1: Prévalence de la lèpre au Togo [Prevalence of leprosy in Togo].

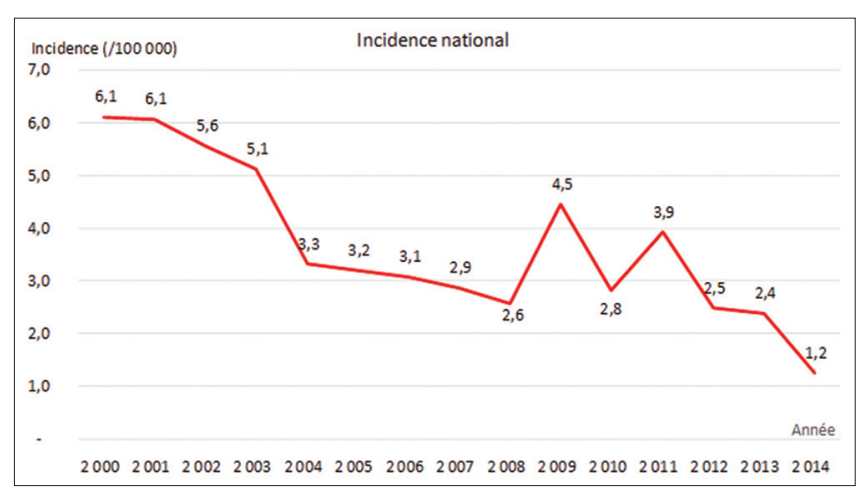

Figure 2: Incidence annuelle des formes clinique de la lèpre [Annual incidence of clinical forms of leprosy].

traitement au moment de l'enquête. La durée moyenne du traitement était de 11 mois. La majorité 1690 (73\%)avaient reçu un traitement PCT pendant moins de 12 mois.

Le degré d'infirmité à l'échelle de l'OMS était de grade 0 chez $72,7 \%$ des patients, grade 1 chez $12,3 \%$ et grade 2 chez 15,0\% des patients.

L'évolution était favorable chez $86,1 \%$ des patients, $101(4,4 \%)$ étaient décédés et 9,5\% étaient perdu de vue.

\section{DISCUSSION}

Le taux d'incidence actuelle de la lèpre au Togo témoigne de son élimination comme problème de santé public. En fin 2015, 2,9 nouveau cas pour 100000 habitants ont été rapportés dans 138 pays de l'ensemble des régions OMS, ceci grâce à la mise à disposition gratuitement de la PCT aux patients infectés. Lélimination de la lèpre à l'échelle mondiale a été atteinte en 2000 d'après les chiffres de l'OMS [5].

Lâge moyen de nos patients était de 41 ans. Ceci se rapproche de l'âge moyen des patients dans une 


\section{Burkina-Faso}

Ghana

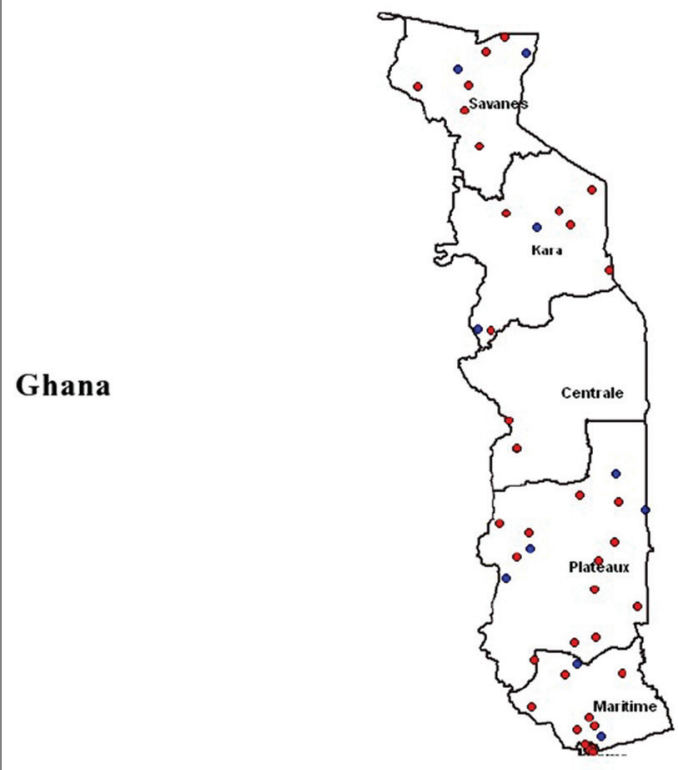

1 point $=50$ cas de $\mathrm{MB}$

01 point $=50$ cas de $\mathrm{PB}$

Bénin

\section{Océan Atlantique}

Figure 3: Cartographie de la lèpre au Togo [Mapping leprosy in Togo].

précédente étude à Lomé qui était de 35 ans. Muthuvel et al en Inde avait trouvé un âge moyen de 32 ans [6] et Keita [7] au Mali avait trouvé un âge moyen de 33,8 ans. Les nouveaux cas de lèpre au Togo sont donc plus fréquents chez l'adulte jeune. Dans notre étude, 5,6\% des nos patients étaient âgés de moins de 15 ans. Ce taux se rapproche des 6,4\% [8] en 2014 au Brésil. Il reste cependant inférieur au $10 \%$ rapporté en 2015 en Inde [6] et 14\% rapporté en 2002 au Sénégal [9]. Dans une précédente étude à Lomé la capitale, 6,4\% des patients étaient âgé de moins de 15 ans. Une diminution de proportion d'enfants de $60 \%$ parmi les nouveau cas de lèpre à été également noté en Ethiopie [10]. Nous pouvons donc conclure à une diminution de l'incidence de la maladie chez les enfants. La proportion des enfants est considérée comme un indicateur de transmission de la maladie, et une diminution du taux signifie une diminution de l'incidence.
Une prédominance masculine de la lèpre est généralement rapportée dans la littérature $[2,6,7,11]$. Notre étude a confirmé cette tendance générale avec un sex-ratio (homme/femme)de 1,13.

Selon les critères OMS, la lèpre est éliminée du Togo depuis 1996 [12]. Notre étude étant une étude rétrospective, il est difficile d'expliquer formellement les variations de taux d'incidence observées entre 2000 et 2014 aux Togo. Mais le dépistage de la lèpre au Togo étant passif, les difficultés d'accès des patients aux structures de soins, les pratiques d'automédication par les populations et l'absence d'activités communautaires peuvent expliquer ces variations de prévalence d'une année à une autre. D'autres facteurs notamment une augmentation de la population dans certaines régions sanitaires du pays, et la fluctuation de la population peuvent expliquer cette variation de taux d'incidence d'une année à une autre. 
La région des plateaux dans le centre du pays cumul l'incidence le plus élevée de la lèpre au cours des 15 dernières années (environs 800 cas). Malgré donc l'élimination de la lèpre au Togo depuis une vingtaine d'année, il persiste des foyers potentiels de la maladie dans certaines régions. D'ou l'importance d'amplifier les activités d'éradications de la maladie dans les villes de ces régions afin d'éviter une recrudescence de la maladie.

Les formes MB étaient les plus fréquentes (74,2\%). Dans une précédente étude à Lomé, la ville capitale du pays, les formes MB représentaient 57,9\%. La forme MB reste celle prédominante dans d'autres études dans plusieurs pays ou l'épidémie à été déclaré ne plus constitué un problème de santé publique. Ainsi, respectivement $60 \%$ et $65 \%$ des cas au Sénégal et en Inde étaient des formes MB [6,9]. D'autres études $[7,13]$ ont montré aussi la survenue d'une inversion de tendance en faveur de la forme MB en rapport avec une diminution de la prévalence. La prédominance de plus en plus des formes MB augmente les risques de contagiosité de la maladie. Aussi, la durée du traitement relativement plus longue des formes $\mathrm{MB}$ peut conduire à l'abandon du traitement par les patients (augmentation des perdus de vue au cours du traitement) et par conséquent contribuer à la propagation de la maladie et la survenue des formes résistante

Parmi les patients inclus, 27,3\% avaient une infirmité grade 1 ou 2 de l'OMS. Ceci témoigne d'un diagnostic assez tardif et/ou une prise en charge retardée par la polychimiothérapie des patients. En 2002 au Sénégal, 14\% des patients avaient une infirmité grade 2 de l'OMS [9].

Lévolution sous chimiothérapie était favorable chez 86,1\% de nos patients; cependant, l'évolution de la maladie était également influencée par la durée de la chimioprophylaxie. En effet, les patients ayant été traité pendant plus de 12 mois avaient une issue favorable

\section{CONCLUSION}

Lincidence de la lèpre dans notre étude témoigne de son élimination comme problème prioritaire de santé publique au Togo. Cependant, la prédominance des formes $\mathrm{MB}$ peut faire craindre à nouveau le risque de recrudescence de la maladie. D’ou la nécessité de faire des campagnes de dépistage actif pour une prise en charge des cas non dépistés.

\section{ACKNOWLEDGEMENTS}

Les auteurs remercient l'Association Allemande de lutte contre la Lèpre et la Tuberculose (DAHW-Togo) pour avoir mis à leur disposition les données nationaux sur la lèpre.

\section{REFERENCES}

1. WHO. lèpre/épidémiologie: le point sur l'épidémiologie de la lèpre dans le monde en 2014 bull. de l'ALLF no 31, juin 2016.

2. Saka B, Kombaté K, Mouhari-Toure A, Amegan-Aho KH, TchangaiWalla K, Pitché P. Lèpre à Lomé (Togo): étude rétrospective de 383 cas. Med Trop. 2008:68:283-6.

3. Ministère de la Planification, du Développement et del'Aménagement du Territoire (MPDAT); Direction Générale de la statistique et de la Comptabilité Nationale (DGSCN)Recensement Général de la Population et de l'Habitat au Togo 2014.

4. WHO. Geneva. A guide to eliminating leprosy as a public health problem. WHO/LEP/95, 1995.

5. WHO. Leprosy Elimination Project. Status report 2016 of. [en ligne] [18.02.17] accessible sur internet. htpp:/www.who.int/lep. Consulter le 20-04-2017.

6. Muthuvel T, Isaakidis P, Shewade HD, Kattuppara L, Singh R, Govindarajulu S. Leprosy trends at a tertiary care hospital in Mumbai, India, from 2008 to 2015 Glob Health Action. 2016;9:32962.

7. Keita S, Tiendrebeogo A, Konaré H, Cissé BS, Faye O. Nouveaux cas de lèpre à l'Institut Marchoux. Etude comparative 1988-1997. Ann Dermatol Venereol. 2001;128:217-9.

8. Nobre ML, Illarramendi X, Dupnik KM, Hacker MA, Nery JA, Jerônimo SM, et al. Multibacillary leprosy by population groups in Brazil: Lessons from an observational study. PloS Negl Trop Dis. 2017;11:e0005364.

9. Seydi M, Mané I, Soumaré M, Faye O, Badiane C, Coly SL, et al. Évaluation de l'endémie lépreuse au Sénégal en 2002. Med Mal Inf. 2005;35:225-7.

10. Groenen G. Trends in prevalence and case finding in the ALERT leprosy control programme, 1979-1999. Lepr Rev. 2002;73:29-40.

11. Peters ES, Eshiet AL. Male-female (sex)differences in leprosy patients in south eastern Nigeria: females present late for diagnosis and treatment and have higher rates of deformity. Lepr Rev. 2002;73:262-7.

12. Programme National de Lutte contre la Lèpre et la TuberculoseTogo. Indicateurs d'élimination de la lèpre au Togo de 1990 à 2005. (Rapports).

13. Ogbeiwi OI. Progress towards the elimination of leprosy in Nigeria: a review of the role of policy implementation and operational factors. Lepr Rev. 2005;76:65-76.

Copyright by Koussake Kombate, et al. This is an open-access article distributed under the terms of the Creative Commons Attribution License, which permits unrestricted use, distribution, and reproduction in any medium, provided the original author and source are credited.

Source of Support: Nil, Conflict of Interest: None declared. 